\title{
Comparative genomic analysis of inbred rat strains reveals the existence of ancestral polymorphisms
}

\author{
Hyeonjeong Kim ${ }^{1} \cdot$ Minako Yoshihara $^{1} \cdot$ Mikita Suyama $^{1}$ D
}

Received: 13 November 2019 / Accepted: 2 March 2020 / Published online: 12 March 2020

(c) The Author(s) 2020

\begin{abstract}
In an alignment of closely related genomic sequences, the existence of discordant mutation sites, which do not reflect the phylogenetic relationship of the genomes, is often observed. Although these discordant mutation sites are thought to have emerged by ancestral polymorphism or gene flow, their frequency and distribution in the genome have not yet been analyzed in detail. Using the genome sequences of all protein coding genes of 25 inbred rat strains, we analyzed the frequency and genome-wide distribution of the discordant mutation sites. From the comparison of different substrains, it was found that these loci are not substrain specific, but are common among different groups of substrains, suggesting that the discordant sites might have mainly emerged through ancestral polymorphism. It was also revealed that the discordant sites are not uniformly distributed along chromosomes, but are concentrated at certain genomic loci, such as $R T 1$, major histocompatibility complex of rats, and olfactory receptors, indicating that genes known to be highly polymorphic tend to have more discordant sites. Our results also showed that loci with a high density of discordant sites are also rich in heterozygous variants, even though these are inbred strains.
\end{abstract}

\section{Introduction}

After genome sequencing had been completed for representative model organisms, such as mice and rats (Waterston et al. 2002; Gibbs et al. 2004), genomes of other species have been sequenced and even more sequencing projects are in progress (Koepfli et al. 2015). At the beginning, genome comparison was conducted to elucidate the genes in a genome, because the basic gene set was thought to be conserved against the intergenic regions in a genome (Brenner et al. 1993). By comparing various genome sequences of different species in a clade, it is also possible to identify functionally important sites other than genes (i.e., conserved non-coding sequences). Not only genome sequence conservation among species but also species-specific presence or

Electronic supplementary material The online version of this article (https://doi.org/10.1007/s00335-020-09831-7) contains supplementary material, which is available to authorized users.

Mikita Suyama

mikita@bioreg.kyushu-u.ac.jp

1 Division of Bioinformatics, Medical Institute of Bioregulation, Kyushu University, Maidashi 3-1-1, Higashi-ku, Fukuoka 812-8582, Japan absence of certain genome sequences have provided insights into the characteristic traits of a species.

In the past decade, genomes of different strains or individuals within a species have been actively sequenced (Saar et al. 2008; Keane et al. 2011; Yalcin et al. 2012; Auton et al. 2015; Hermsen et al. 2015).Technically, this was facilitated for two main reasons. One reason is that, if the reference genome for a species has already been determined, genome sequencing for other strains for the species is comparatively easy because laborious assembling process is not required. The other reason is that next-generation sequencing technology, which is still drastically improving in terms of not only throughput but also time and cost, is greatly accelerating research in this direction. By comparing these closely related genome sequences, we can identify strain-specific traits such as disease susceptibility (e.g., Fairfield et al. 2011).

Rats are one of the species for which the genome sequence information of various strains is available (Saar et al. 2008; Hermsen et al. 2015). Compared to mice, in which genetic manipulation, such as gene knockout techniques, is well developed, rats have been, until recently, inferior with regard to genetic analyses, although rats are well suited, for example, for transplantation experiments and behavioral analyses because of their body size and obedient nature. The advent of genome editing technologies, however, 
has enabled the genetic manipulation of rats (Mashimo et al. 2013), and genetic analyses in rats will be greatly advanced in the future. Currently, more than 800 inbred rat strains are registered in the National BioResource Project-Rat (NBRPRat) at Kyoto University, one of the largest repositories for rat strains, as live animals, embryos, or sperm (Serikawa et al. 2009), and we have determined the protein coding genes and non-coding conserved sequences of some representative rat strains registered in NBRP-Rat (Yoshihara et al. 2016a, b; Kuramoto et al. 2017).

From the genome sequence comparison of such closely related strains, it is possible not only to identify strainspecific mutations to explain a certain trait for the strain, based on the differences in the genomic sequences, but also to conduct a large-scale analysis of mutation patterns among strains. A discordant mutation is one such mutation pattern, in which the pattern of base changes at a certain site in the genome is not consistent with the branching topology of the phylogenetic tree of strains based on the total number of variations among the strains (Fitch 1977). These discordant mutation patterns are thought to be observed if ancestral polymorphism or gene flow exists in a certain group of strains (Liu et al. 2008). Although it is possible to infer the reason why such mutation sites have emerged, their frequency and distribution in the genome are largely unknown.

In this study, using the genomic sequences of all protein coding genes of 25 inbred rat strains that we recently sequenced (Yoshihara et al. 2016a, b; Kuramoto et al. 2017), we first analyzed the phylogenetic relationship among them, and then comprehensively identified variant sites that showed discordant mutation patterns. We found that discordant sites are not uniformly distributed along chromosomes, but are concentrated at certain genomic loci. From detailed analyses, it is suggested that the discordant sites might have emerged mainly through ancestral polymorphism, and the loci are still rich in heterozygous variants, even though these are inbred strains.

\section{Materials and methods}

\section{Data set}

We used the genomic sequences corresponding to the coding regions of 25 inbred rat strains (DDBJ Sequence Read Archive accession number: DRA004543; DDBJ/EMBL/ GenBank accession number: PRJDB4648) obtained from our previous studies (Yoshihara et al. 2016a, b; Kuramoto et al. 2017) (Table 1). These 25 strains were selected according to the following three categories: representative inbred strains (F344/DuCrlCrlj, F344/Jcl, F344/NSlc, and F344/ $\mathrm{Stm})$, those originating from wild populations (BN/SsNSlc, DOB/Oda, IS/Kyo, IS-Tlk/Kyo, LE/Stm, LEC/Tj, and
Table 1 List of 25 rat strains analyzed in this study

\begin{tabular}{|c|c|c|c|}
\hline Rat no & Strain name & Inbred generations $^{\mathrm{a}}$ & $\overline{\text { NBRP no }}$ \\
\hline 1 & BDIX.Cg-Tal/NemOda & - & 0305 \\
\hline 2 & BDIX/NemOda & F11 (March 2012) & 0304 \\
\hline 3 & $\mathrm{BN} / \mathrm{SsNSlc}$ & - & 0149 \\
\hline 4 & BUF/Mna & F122 (April 2012) & 0200 \\
\hline 5 & DOB/Oda & F29 (April 2012) & 0307 \\
\hline 6 & F344/DuCrlCrlj & - & 0506 \\
\hline 7 & F344/Jcl & - & None \\
\hline 8 & F344/NSlc & F188 & 0156 \\
\hline 9 & F344/Stm & F91 (April 2012) & 0140 \\
\hline 10 & HTX/Kyo & F? + 54 (March 2012) & 0006 \\
\hline 11 & HWY/Slc & $\mathrm{F} ?+11$ & 0152 \\
\hline 12 & IS/Kyo & F88 (March 2012) & 0008 \\
\hline 13 & IS-Tlk/Kyo & F65 (May 2009) & 0009 \\
\hline 14 & KFRS3B/Kyo & - & 0571 \\
\hline 15 & KFRS4/Kyo & F24 (April 2012) & 0572 \\
\hline 16 & $\mathrm{LE} / \mathrm{Stm}$ & F111 (April 2012) & 0139 \\
\hline 17 & $\mathrm{LEC} / \mathrm{Tj}$ & F100 & 0051 \\
\hline 18 & NER/Kyo & F70 (March 2012) & 0010 \\
\hline 19 & NIG-III/Hok & F140 (April 2012) & 0044 \\
\hline 20 & PVG/Seac & F59 (April 2012) & 0080 \\
\hline 21 & RCS/Kyo & F47 (March 2012) & 0011 \\
\hline 22 & WTC/Kyo & F86 (March 2012) & 0020 \\
\hline 23 & WTC-Swh/Kyo & F37 (March 2012) & 0287 \\
\hline 24 & $\mathrm{ZF}$ & - & None \\
\hline 25 & ZFDM & - & None \\
\hline
\end{tabular}

Information obtained from the NBPR-Rat web site (https://www. anim.med.kyoto-u.ac.jp/NBR/). The inbred generation represents the generation at the time point shown in the parentheses. For strains whose time point is not provided, it is not written. Question mark ("?") indicates that the number of inbred generations before transfer to NBRP-Rat is unknown. Hyphen ("-") indicates that the information about inbred generations was not available

NIG-III/Hok), and disease models derived from selective breeding (BDIX/NemOda, BDIX.Cg-Tal/NemOda, BUF/ MNa, HTX/Kyo, HWY/Slc, KFRS3B/Kyo, KFRS4/Kyo, NER/Kyo, PVG/Seac, RCS/Kyo, WTC/Kyo, WTC-swh/ Kyo, ZF, and ZFDM). All these strains are kept in NBRPRat, Kyoto University (Kyoto, Japan) (Serikawa et al. 2009). We downloaded rat genome rn5 (RGSC 5.0, March 2012) from Ensembl (ftp://ftp.ensembl.org/pub/release-79/fasta/ rattus_norvegicus/dna/) (Zerbino et al. 2018) and used this as a reference genome.

\section{Identification and annotation of variants}

The genomic sequence data corresponding to the coding regions were processed as reported in our previous study (Yoshihara et al. 2016a, b). In brief, the sequencing reads were mapped to the rat reference genome (rn5) using BWA 
(v.0.7.4) (Li and Durbin 2009) with the default parameters. SAMtools (v.0.1.12a) (Li et al. 2009; Li 2011), Picard Tools (v.1.87) (https://broadinstitute.github.io/picard/), and the Genome Analysis Toolkit (GATK, v.2.5.2) (McKenna et al. 2010) were used for post-processing of mapped reads. The UnifiedGenotyper utility in GATK was used for variant calling. Since there is a risk of misalignment of sequencing reads and collapsed mapping of sequencing reads, we used only homozygous variants, but no heterozygous variants in this study unless otherwise noted. The details of these conditions are illustrated in "Discussion". ANNOVAR (version 2015-03-22) (Wang et al. 2010) was used to annotate these variants.

\section{Construction of a phylogenetic tree}

Phylogenetic trees were constructed in the following three steps. (1) The distance matrix was cleated based on SNV data. (2) The distance matrix was supplied to the "ape" package (v.5.0) (Paradis and Schliep 2019) in R (v.3.5.1) (The R Project for Statistical Computing, Vienna, Austria) to create a phylogenetic tree based on the neighbor-joining method (Saitou and Nei 1987). The data of the phylogenetic tree was obtained in Newick format. (3) To visualize the Newick-formatted file obtained above, a phylogenetic tree was depicted by the Dendroscope 3 program (v.3.5.10) (Huson and Scornavacca 2012).

\section{Identification of discordant sites}

To automatically identify discordant sites, we first generated multiple genome sequence alignment of the coding DNA sequences (CDSs) by reflecting the SNV data into the rat reference sequence. Information about CDSs was obtained from the Ensembl (Zerbino et al. 2018) and RefSeq (O'Leary et al. 2016) annotations in the UCSC Genome Browser (Casper et al. 2018). Then, discordant sites were identified as homozygous variant sites in the genome sequence alignment that showed patterns of mutation inconsistent with the branching order in the phylogenetic tree. More precisely, at a variant site in a closely related substrain cluster, if the same variation is also observed in other strains, which are thought to be more distantly related to those in the substrain cluster, we defined such a site as a discordant site. The examples of the discordant sites are illustrated in "Results". At this step, we used the "tree" module of the ETE Toolkit 3 (v.3.1.1) (Huerta-Cepas et al. 2016) to analyze the branching pattern of the phylogenetic tree in Newick format. The alignments were visualized using Jalview (v.2) (Waterhouse et al. 2009).

To analyze the distribution of discordant sites along chromosomes, information on the chromosome lengths and cytogenetic bands of rats (rn5) was downloaded from the UCSC Genome Browser (https://hgdownload.cse.ucsc.edu/
goldenPath/rn5/bigZips/rn5.chrom.size and https://hgdow nload.cse.ucsc.edu/goldenPath/rn5/database/cytoBand.txt. gz, respectively). The graph of the chromosomal distribution of the discordant sites was created using the "ggplot2" package (Wickham 2016) of the R software.

\section{Enrichment analysis of genes}

For enrichment analysis of genes, we used Metascape (Zhou et al. 2019). We set both "Input as species" and "Analysis as species" options to " $R$. norvegicus."

\section{Results}

\section{Identification of coding variants in each strain}

First, we identified homozygous coding variants in the 25 inbred rat strains. This analysis was performed in comparison with the reference genome, which is determined for Brown Norway rats (Gibbs et al. 2004) (Table 1). The variants were then classified by applying ANNOVAR (Wang et al. 2010). In each strain, the number of synonymous variants was approximately twice that of non-synonymous variants. The number of stop-gain mutations was approximately 10 times as much as the number of stop-loss mutations (Tables 2, S1), showing a similar ratio to that reported in a large-scale mutational analysis in cattle (Charlier et al. 2016). The highest number of mutations was observed in the DOB/Oda strain, which is a Japanese wild-derived rat strain (Kuramoto et al. 2013).

\section{Phylogenetic trees based on exome target capture SNV data}

To reveal the genetic relationship of the 25 inbred rat strains (Table 1), we attempted to construct a phylogenetic tree. For this, we prepared a distance matrix that summarized the genetic distances of all possible pairs of strains. Here, the genetic distances are calculated on the basis of the information about the mutations compared to the reference genome. For example, in calculating the number of mutations between strains A and B from the information about the mutations of strain $A$ versus the reference genome and strain B versus the reference genome, we enumerated only those mutations that can be observed either in strain $A$ or in strain B but not in both strains. Then, the obtained distance matrix was subjected to the neighbor-joining algorithm to construct an unrooted phylogenetic tree (Fig. 1). As DOB/ Oda is a wild-derived rat, it is clearly separated from the other laboratory rat strains (Kuramoto et al. 2013). A moderate similarity between PVG/Seac and KFRS4/Kyo well reflects the origin of the KFRS4/Kyo strain; that is, the strain 
Table 2 The numbers of synonymous, non-synonymous, stop-gain, and stop-loss SNVs in the 25 rat strains

\begin{tabular}{|c|c|c|c|}
\hline Strain name & Synonymous SNV & $\begin{array}{l}\text { Non-syn- } \\
\text { onymous } \\
\text { SNV }\end{array}$ & Stop-gain/loss \\
\hline $\begin{array}{l}\text { BDIX.Cg-Tall } \\
\text { NemOda }\end{array}$ & 9006 & 4777 & $25 / 1$ \\
\hline BDIX/NemOda & 8659 & 4565 & $19 / 2$ \\
\hline $\mathrm{BN} / \mathrm{SsNSlc}$ & 113 & 193 & $3 / 0$ \\
\hline BUF/Mna & 8518 & 4777 & $22 / 3$ \\
\hline DOB/Oda & 11,042 & 5810 & $28 / 2$ \\
\hline F344/DuCrlCrlj & 8396 & 4601 & $20 / 1$ \\
\hline $\mathrm{F} 344 / \mathrm{Jcl}$ & 8283 & 4538 & $22 / 1$ \\
\hline F344/NSlc & 8294 & 4548 & $19 / 1$ \\
\hline F344/Stm & 8379 & 4547 & $20 / 1$ \\
\hline НTX/Куо & 8496 & 4741 & $17 / 4$ \\
\hline HWY/Slc & 8680 & 4609 & $18 / 2$ \\
\hline IS/Kyo & 10,585 & 5820 & $31 / 2$ \\
\hline IS-Tlk/Kyo & 10,596 & 5819 & $30 / 2$ \\
\hline KFRS3B/Kyo & 8440 & 4480 & $21 / 2$ \\
\hline KFRS4/Kyo & 9019 & 5049 & $18 / 5$ \\
\hline $\mathrm{LE} / \mathrm{Stm}$ & 8545 & 4558 & $18 / 4$ \\
\hline $\mathrm{LEC} / \mathrm{Tj}$ & 9389 & 5103 & $26 / 3$ \\
\hline NER/Kyo & 7140 & 3829 & $11 / 3$ \\
\hline NIG-III/Hok & 9150 & 4960 & $23 / 3$ \\
\hline PVG/Seac & 9113 & 5013 & $21 / 4$ \\
\hline RCS/Kyo & 8421 & 4594 & $24 / 4$ \\
\hline WTC/Kyo & 10,017 & 5713 & $28 / 3$ \\
\hline WTC-Swh/Kyo & 10,018 & 5724 & $29 / 3$ \\
\hline $\mathrm{ZF}$ & 8395 & 4517 & $18 / 2$ \\
\hline ZFDM & 8486 & 4623 & $18 / 2$ \\
\hline
\end{tabular}

is an inbred one derived from the crossing of a fancy rat with the PVG/Seac strain (Kuramoto et al. 2010). Using the same procedure, the phylogenetic tree was also made for each of the 20 rat autosomes (Supplementary Fig. S1). The percentage values on branches represent the branch support value at the chromosome level (Fig. 1). For example, $80 \%$ means that the phylogeny of 16 out of 20 autosomes has the same internal branch as the corresponding one in the phylogeny of all the chromosomes. There are clusters with a high branch support value, each of which is comprised of substrains: BDIX (BDIX/NemOda and BDIX.Cg-Tal/NemOda), F344 (F344/DuCrlCrlj, F344/Jcl, F344/NS1, and F344/Stm), IS (IS-Tlk/Kyo and IS/Kyo), WTC (WTC/Kyo and WTC-swh/ $\mathrm{Kyo}$ ), and ZF (ZF and ZFDM). In the phylogenetic tree for chromosome 20 (Supplementary Fig. S1), the LE/Stm strain is not directly clustered with the LEC/Tj strain, but is clustered with BN/ScNSlc and the BDIX cluster. The substrains in the ZF cluster, ZF and ZFDM, are also separated in the phylogenetic tree for chromosome 20 . Such instability in branching might be due to the relatively small number of mutations on the relatively short chromosome 20 . Separation of ZF substrains was also observed in the phylogenetic tree for chromosome 16 (Supplementary Fig. S1).

\section{Discordant sites among 25 rat strains}

By looking at multiple genome sequence alignments of these strains in detail, we were able to easily find variant sites that showed patterns of mutation inconsistent with the branching order in the phylogenetic tree constructed from the number of mutations in the coding regions. For example, variants observed in the cluster of $\mathrm{ZF}$, which consists of the ZF and ZFDM strains, also existed in the other strains, despite their
Fig. 1 Phylogenetic tree of 25 inbred rat strains. This tree was obtained using the neighborjoining algorithm based on SNV data of all the coding regions. The value at each internal branch indicates the branch support value as a percentage for chromosome level. Only values greater than $50 \%$ are shown. The scale bar at the bottom left indicates one nucleotide substitution per 10,000 bases

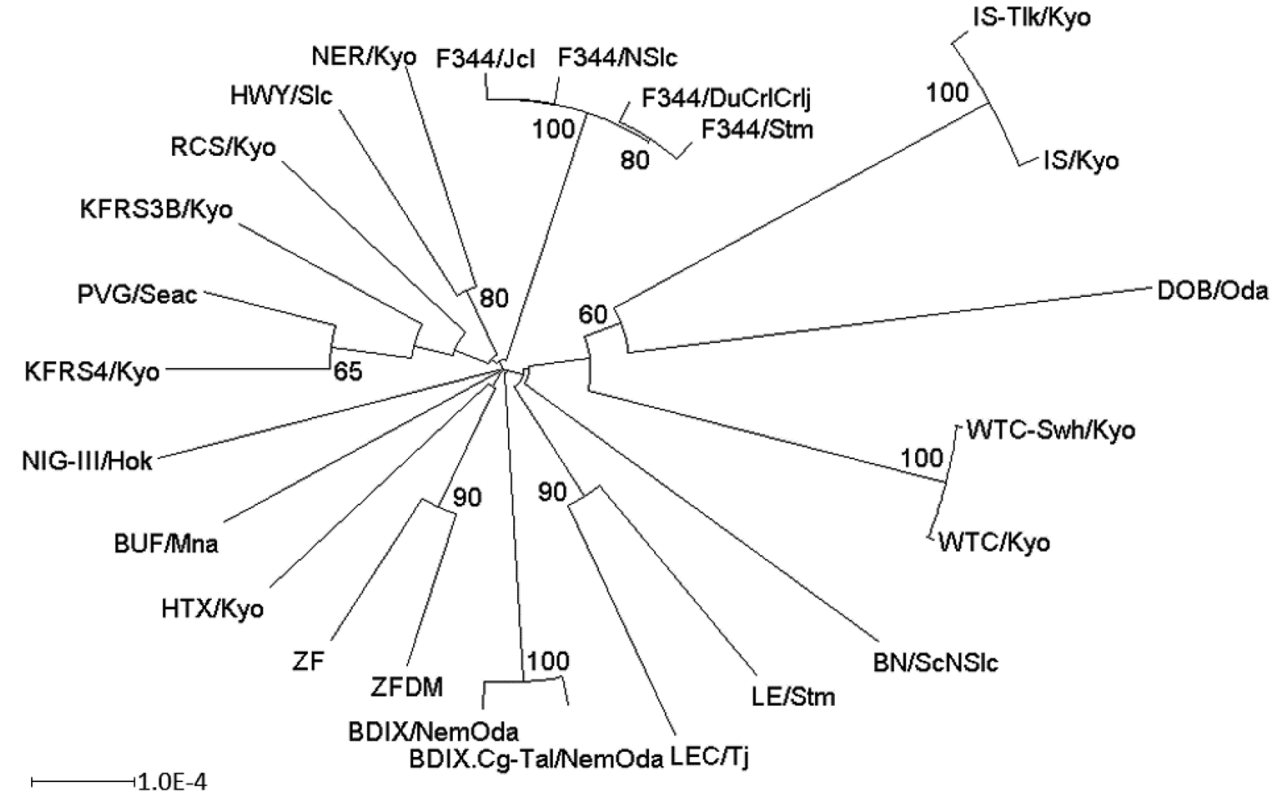


distance measured by the number of mutations in coding regions (Fig. 2a). Hereafter, we define such a variant site, i.e., the site with variants in a cluster of closely related substrains also existing in the other strains, as a "discordant site." Discordant sites are also observed in another cluster of closely related substrains, such as the cluster of F344 (Fig. 2b), and in other genes (Supplementary Fig. S2).

To quantitatively assess the occurrence of discordant sites, we counted the number of discordant sites for each cluster of substrains (Tables 3, S2). The number of discordant sites is not uniform among the five clusters of substrains, but varies from 234 (for WTC) to 6146 (for ZF). This difference may be attributed to the divergence of the substrains in each cluster; that is, a more diverged cluster, such as ZF, tends to have a higher content of discordant sites, whereas a less diverged cluster, such as WTC, tends to have a lower content of discordant sites.

To analyze the distribution of the discordant sites along chromosomes, those sites were visualized by displaying them on bar graphs that represented ideograms of
Table 3 The number of discordant sites in the CDS of five clusters of substrains (BDIX, F344, IS, WTC, and ZF)

rat chromosomes (Fig. 3). Although discordant sites are observed all over the chromosomal positions, they are not uniformly distributed, but have a clear trend of aggregating at certain genomic locations. Moreover, the positions of these genomic regions, which have a relatively high number of discordant sites, are shared among different clusters of substrains (Fig. 3). For example, highly dense regions of discordant sites in the $\mathrm{p}$-arm of chromosome 20 found in the BDIX substrains (Fig. 3a) are also found in the WTC
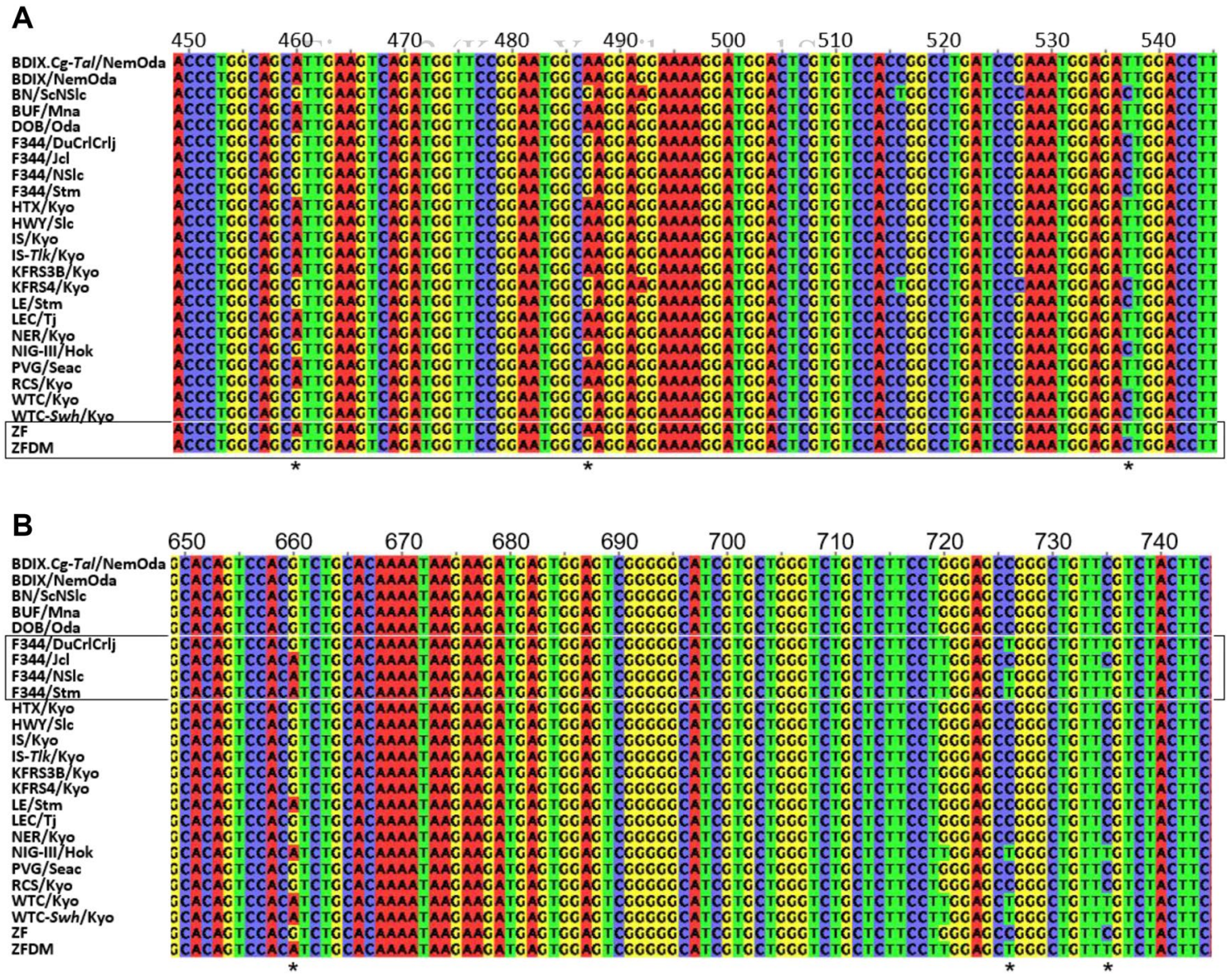

Fig. 2 Examples of discordant sites in multiple sequence alignments of $R T 1-D b 1$ gene on 20p12. The box indicates the cluster of substrains. The numbers above the alignment indicate the positions in
mRNA. Discordant sites are indicated by asterisks under the alignments. a Discordant sites observed in the cluster of ZF substrains. $\mathbf{b}$ Discordant sites observed in the cluster of F344 substrains 

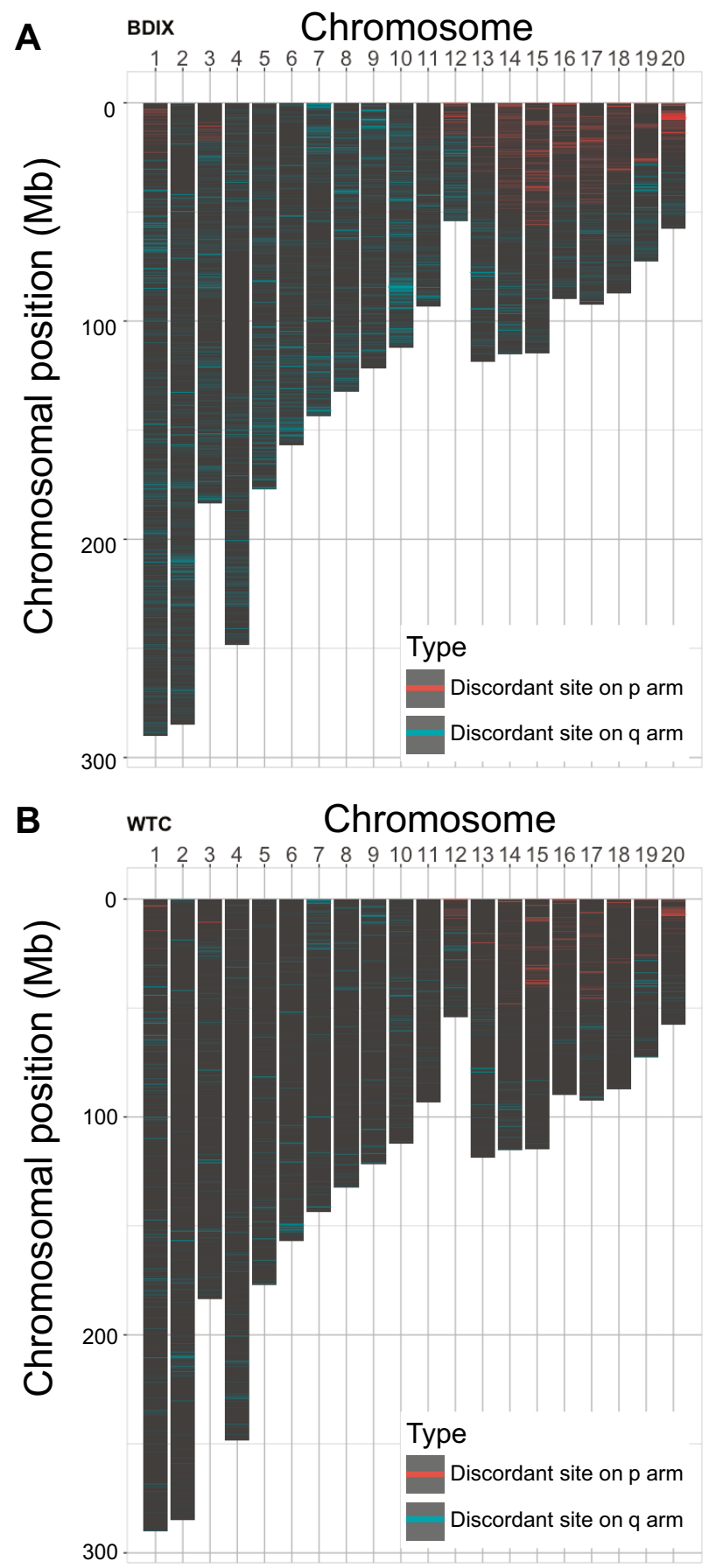

Fig. 3 Chromosomal distribution of discordant sites in the autosomes of the clusters of a BDIX and $\mathbf{b}$ WTC substrains. The red and blue horizontal lines on the chromosomes represent discordant sites in the short (p) arm and long (q) arm, respectively

substrains (Fig. 3b). The overall pattern of the distribution of discordant sites is also similar in other clusters of substrains (Supplementary Fig. S3), indicating the existence of certain characteristics for such regions.

\section{Enrichment analysis of discordant site abundant genes}

To comprehensively analyze the characteristics of regions with a relatively high number of discordant sites, we first extracted genes with discordant sites and sorted them according to the density of the discordant sites. There were 6,216 genes with at least one common discordant site in the five clusters of substrains. The gene with the highest density of discordant sites was $R T 1$, which is a major histocompatibility complex (MHC) of rats, located at 20p12 (Aptekman 1960; Günther and Walter 2001).

The top 500 genes with a high density of discordant sites were subjected to a functional enrichment analysis by Metascape (Zhou et al. 2019) (Supplementary Table S3). The result showed that discordant site-rich genes tend to be immune-related genes and olfactory receptor genes (Fig. 4). As the cutoff, the top 500, was arbitrarily selected, we tried some other cutoff values, that is, a standard deviation value of the discordant site content, which yielded 1,176 genes, and the mean value of the discordant site content, which yielded 2,035 genes (Supplementary Fig. S4). The functional enrichment analysis of these lists of genes also yielded similar results to that of the top 500 genes (Supplementary Fig. S5).

\section{Correspondence between discordant site-rich regions and heterozygous variant-rich regions}

Discordant sites are concentrated on the genes that tend to be polymorphic, such as immune-related genes and genes involved in sensory signaling pathways. Although this might be reflected by a high level of polymorphism in their ancestors, the strains in the present state still might have a certain degree of polymorphism in the same regions, even though they are inbred strains. To confirm this, we enumerated the number of heterozygous sites. They have heterozygous sites up to $0.02 \%$ (in WTC-Swh/Kyo), this is comparable to what is expected for the inbred generations (F37) (Table 1). We did not observe any correlation between inbred generations and proportion of heterozygous variants. We plotted these heterozygous variations along the chromosomes and compared the distribution with that of the discordant sites (Fig. 5). Indeed, both distributions correspond well with each other, indicating that the regions where discordant sites are concentrated still have heterozygous sites.

\section{Discussion}

Inbred strains are a valuable resource for analyzing the genetic bases of strain-specific traits. Comparative genomic sequence analysis among strains is a fundamental step for 


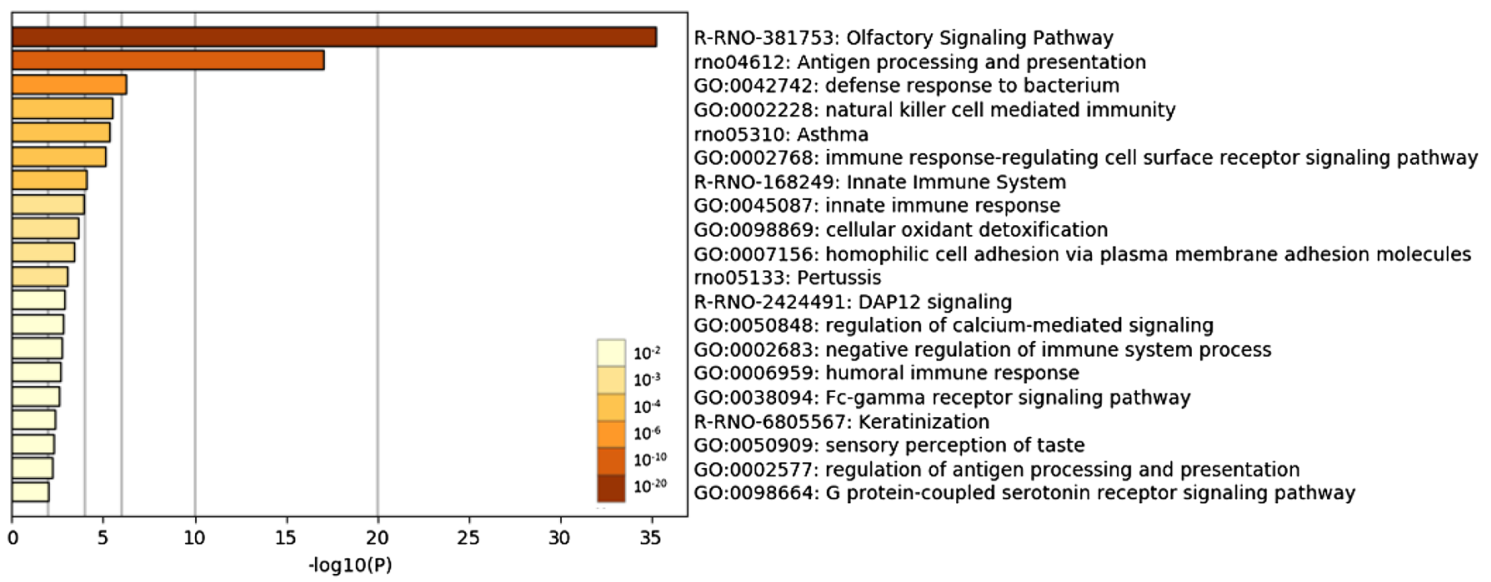

Fig. 4 Bar graph of the functional enrichment analysis by Metascape. The enrichment analysis was performed using the top 500 genes from the list of genes with discordant sites. The bars are colored by $P$-val-

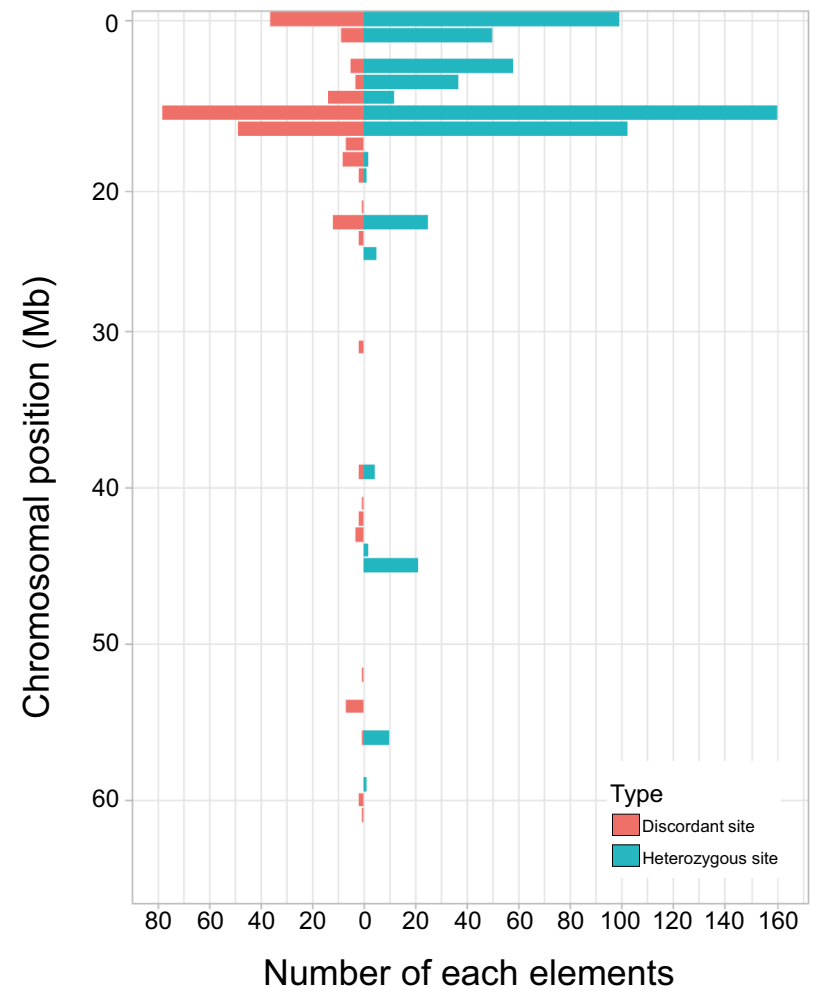

Fig. 5 Distributions of discordant sites and heterozygous SNVs. The number of discordant sites and heterozygous SNVs in the F344 are shown in orange and blue, respectively, on chromosome 20

identifying genomic sequence segments responsible for certain traits and also for understanding the mode of genomic sequence divergence. For this, we conducted a comparative genomic sequence analysis of coding regions of 25 inbred rat strains, focusing on discordant sites, which show an ues. GO, gene ontology; rno, KEGG pathway for rats; R-RNO, Reactome gene sets for rats

inconsistency between genetic relatedness of strains and patterns of sequence variations. We found that such sites exist all over the chromosomes. They are not uniformly distributed along chromosomes, but are concentrated on the loci comprising immunity and sensory genes. In addition, we found that regions rich in discordant sites are also rich in heterozygous variants, although these strains are thought to be genetically homozygous for most of the loci because of inbreeding.

There are at least two possible explanations for the emergence of discordant sites. One possibility is the existence of ancestral polymorphisms (Slatkin and Pollack 2008). This can be explained as follows: during the establishment process for each inbred strain, polymorphic loci that existed in their ancestral strain have randomly fixed to a single allele. The other possibility is the result of gene flow (Slatkin 1987). Genomic segments that migrated from other strains could have brought variants that are inconsistent with the strains' phylogenetic relationships. However, because gene flow is a stochastic process, it is not likely to happen in certain loci in multiple independent clusters of substrains. Accordingly, we concluded that discordant sites would have originated from polymorphic loci that existed in their nearest common ancestor. This idea can be supported by the fact that such genomic regions contain immunity and sensory genes, such as $R T 1$ and olfactory receptors, which are known to be highly polymorphic (Ehlers et al. 2000; Takagi et al. 2009). In addition, heterozygous variants are also enriched in those loci, indicating that such loci still have polymorphisms even though these are inbred strains. This observation further supports the idea that the discordant sites have emerged by ancestral polymorphisms.

A possible pitfall in the present study is that there could be a misalignment of sequence reads in genomic regions 
with very high similarities. If this happens, we may have false variants (Supplementary Fig. S6a). To prevent such instances, we adopted stringent criteria for read alignment, i.e., we used only those reads that were uniquely mapped to the reference genome with two or fewer mismatches. However, even using only uniquely mapped reads, we could still have false variants by copy number changes between the reference genome and the genome of a strain under consideration (Keane et al. 2011; Doran et al. 2016; Ramdas et al. 2019). For example, if there is a duplicated region in the genome of a strain under analysis, while the reference genome has only a single copy, then the sequencing reads that come from the duplicated region are forced to map to a single locus of the reference genome (Supplementary Fig. S6b). In this case, any differences between the duplicated regions should be detected as variants. To prevent such false variants, we only used homozygous variants for discordant site detection because such collapsed read mapping should result in heterozygous variants (Ramdas et al. 2019). With these conditions, we reliably obtained more high-quality alignments and variants.

In summary, we identified discordant sites by comparing the phylogenetic trees of inbred strains and each position in the genome alignment. Their emergence can be attributed to ancestral polymorphisms because of their enrichment in highly polymorphic loci, such as $R T I$ (Takagi et al. 2009). These regions seem to be still heterozygous to some extent because heterozygous sites are also enriched in discordant site-rich regions. These findings are concordant with some previous reports for other inbred species (Lilue et al. 2018; Wang et al. 2019), providing valuable insights for understanding the genetic characteristics and diversity in highly polymorphic loci in the process of inbreeding. Indeed, in the process of naturally occurring inbreeding, such loci are thought to be under balancing selection and are shown to be heterozygous (Sato et al. 2002; Aguilar et al. 2004; Lins et al. 2018). Our results, together with the findings in other previously reported species (Lilue et al. 2018; Wang et al. 2019), suggest that balancing selection may also act on such loci in the process of artificial inbreeding.

Acknowledgements We would like to thank Hideki Yokoi and Takashi Kuramoto for the valuable discussion and comments on this study. This work was supported in part by the Genome Information Upgrading Program of the National BioResource Project, Japan Agency for Medical Research and Development (AMED) (15km0210068j0001), and a Grant-in-Aid for Scientific Research from the Ministry of Education, Culture, Sports, Science and Technology of Japan (17H03619) to MS.

\section{Compliance with ethical standards}

Conflict of interest The authors declare that they have no conflict of interest.
Open Access This article is licensed under a Creative Commons Attribution 4.0 International License, which permits use, sharing, adaptation, distribution and reproduction in any medium or format, as long as you give appropriate credit to the original author(s) and the source, provide a link to the Creative Commons licence, and indicate if changes were made. The images or other third party material in this article are included in the article's Creative Commons licence, unless indicated otherwise in a credit line to the material. If material is not included in the article's Creative Commons licence and your intended use is not permitted by statutory regulation or exceeds the permitted use, you will need to obtain permission directly from the copyright holder. To view a copy of this licence, visit http://creativecommons.org/licenses/by/4.0/.

\section{References}

Aguilar A, Roemer G, Debenham S et al (2004) High MHC diversity maintained by balancing selection in an otherwise genetically monomorphic mammal. Proc Natl Acad Sci USA 101:34903494. https://doi.org/10.1073/pnas.0306582101

Aptekman AB (1960) The R-l factor, a histocompatibility antigen in the rat. Cancer Res 20:1372-1383

Auton A, Abecasis GR, Altshuler DM et al (2015) A global reference for human genetic variation. Nature 526:68-74. https://doi. org/10.1038/nature 15393

Brenner S, Elgar G, Sanford R et al (1993) Characterization of the pufferfish (Fugu) genome as a compact model vertebrate genome. Nature 366:265-268. https://doi.org/10.1038/366265a0

Casper J, Zweig AS, Villarreal C et al (2018) The UCSC Genome Browser database: 2018 update. Nucleic Acids Res 46:D762D769. https://doi.org/10.1093/nar/gkx1020

Charlier C, Li W, Harland C et al (2016) NGS-based reverse genetic screen for common embryonic lethal mutations compromising fertility in livestock. Genome Res 26:1333-1341. https://doi. org/10.1101/gr.207076.116

Doran AG, Wong K, Flint J et al (2016) Deep genome sequencing and variation analysis of 13 inbred mouse strains defines candidate phenotypic alleles, private variation and homozygous truncating mutations. Genome Biol 17:167. https://doi.org/10.1186/ s13059-016-1024-y

Ehlers A, Beck S, Forbes SA et al (2000) MHC-linked olfactory receptor loci exhibit polymorphism and contribute to extended HLA/OR-haplotypes. Genome Res 10:1968-1978. https://doi. org/10.1101/gr.10.12.1968

Fairfield H, Gilbert GJ, Barter M et al (2011) Mutation discovery in mice by whole exome sequencing. Genome Biol 12(9):R86. https://doi.org/10.1186/gb-2011-12-9-r86

Fitch WM (1977) On the problem of discovering the most parsimonious tree. Am Nat 111:223-257. https://doi.org/10.1086/283157

Gibbs RA, Weinstock GM, Metzker ML et al (2004) Genome sequence of the Brown Norway rat yields insights into mammalian evolution. Nature 428:493-520. https://doi.org/10.1038/ nature 02426

Günther E, Walter L (2001) The major histocompatibility complex of the rat (Rattus norvegicus). Immunogenetics 53:520-542. https ://doi.org/10.1007/s002510100361

Hermsen R, de Ligt J, Spee W et al (2015) Genomic landscape of rat strain and substrain variation. BMC Genom 16:357. https://doi. org/10.1186/s12864-015-1594-1

Huerta-Cepas J, Serra F, Bork P (2016) ETE 3: reconstruction, analysis, and visualization of phylogenomic data. Mol Biol Evol 33:1635-1638. https://doi.org/10.1093/molbev/msw046

Huson DH, Scornavacca C (2012) Dendroscope 3: an interactive tool for rooted phylogenetic trees and networks. Syst Biol 61:10611067. https://doi.org/10.1093/sysbio/sys062 
Keane TM, Goodstadt L, Danecek P et al (2011) Mouse genomic variation and its effect on phenotypes and gene regulation. Nature 477:289-294. https://doi.org/10.1038/nature10413

Koepfli K-P, Paten B, O’Brien SJ (2015) The genome 10K project: a way forward. Annu Rev Anim Biosci 3:57-111. https://doi. org/10.1146/annurev-animal-090414-014900

Kuramoto T, Inoue S, Neoda Y et al (2013) Genetic and phenotypic characterization of a Japanese wild-derived DOB/Oda rat strain. Mamm Genome 24:303-308. https://doi.org/10.1007/ s00335-013-9465-z

Kuramoto T, Voigt B, Nakanishi S et al (2017) Identification of candidate genes for generalized tonic-clonic seizures in noda epileptic rat. Behav Genet 47:609-619. https://doi.org/10.1007/ s10519-017-9870-2

Kuramoto T, Yokoe M, Yagasaki K et al (2010) Genetic analyses of fancy rat-derived mutations. Exp Anim 59:147-155. https://doi. org/10.1538/expanim.59.147

Li H (2011) A statistical framework for SNP calling, mutation discovery, association mapping and population genetical parameter estimation from sequencing data. Bioinformatics 27:2987-2993. https://doi.org/10.1093/bioinformatics/btr509

Li H, Durbin R (2009) Fast and accurate short read alignment with Burrows-Wheeler transform. Bioinformatics 25:1754-1760. https ://doi.org/10.1093/bioinformatics/btp324

Li H, Handsaker B, Wysoker A et al (2009) The Sequence Alignment/ Map format and SAMtools. Bioinformatics 25:2078-2079. https ://doi.org/10.1093/bioinformatics/btp352

Lilue J, Doran AG, Fiddes IT et al (2018) Sixteen diverse laboratory mouse reference genomes define strain-specific haplotypes and novel functional loci. Nat Genet 50:1574-1583. https://doi. org/10.1038/s41588-018-0223-8

Lins LSF, Trojahn S, Sockell A et al (2018) Whole-genome sequencing reveals the extent of heterozygosity in a preferentially selffertilizing hermaphroditic vertebrate. Genome 61:241-247. https ://doi.org/10.1139/gen-2017-0188

Liu Y-H, Takahashi A, Kitano T et al (2008) Mosaic genealogy of the Mus musculus genome revealed by 21 nuclear genes from its three subspecies. Genes Genet Syst 83(1):77-88. https://doi. org/10.1266/ggs.83.77

Mashimo T, Kaneko T, Sakuma T et al (2013) Efficient gene targeting by TAL effector nucleases coinjected with exonucleases in zygotes. Sci Rep 3:1253. https://doi.org/10.1038/srep01253

McKenna A, Hanna M, Banks E et al (2010) The genome analysis toolkit: a MapReduce framework for analyzing next-generation DNA sequencing data. Genome Res 20:1297-1303. https://doi. org/10.1101/gr.107524.110

O'Leary NA, Wright MW, Brister JR et al (2016) Reference sequence (RefSeq) database at NCBI: current status, taxonomic expansion, and functional annotation. Nucleic Acids Res 44:D733-D745. https://doi.org/10.1093/nar/gkv1189

Paradis E, Schliep K (2019) ape 5.0: an environment for modern phylogenetics and evolutionary analyses in R. Bioinformatics 35:526528. https://doi.org/10.1093/bioinformatics/bty633

Ramdas S, Ozel AB, Treutelaar MK et al (2019) Extended regions of suspected mis-assembly in the rat reference genome. Sci data 6:39. https://doi.org/10.1038/s41597-019-0041-6

Saar K, Beck A, Bihoreau MT et al (2008) SNP and haplotype mapping for genetic analysis in the rat. Nat Genet 40:560-566. https://doi. org/10.1038/ng.124
Saitou N, Nei M (1987) The neighbor-joining method: a new method for reconstructing phylogenetic trees. Mol Biol Evol 4:406-425. https://doi.org/10.1093/oxfordjournals.molbev.a040454

Sato A, Satta Y, Figueroa F et al (2002) Persistence of Mhc heterozygosity in homozygous clonal killifish, rivulus marmoratus: implications for the origin of hermaphroditism. Genetics 162(4):1791-1803

Serikawa T, Mashimo T, Takizawa A et al (2009) National BioResource project-rat and related activities. Exp Anim 58(4):333-341. https://doi.org/10.1538/expanim.58.333

Slatkin M (1987) Gene flow and the geographic structure of natural populations. Science 236:787-792. https://doi.org/10.1126/scien ce. 3576198

Slatkin M, Pollack JL (2008) Subdivision in an ancestral species creates asymmetry in gene trees. Mol Biol Evol 25:2241-2246. https ://doi.org/10.1093/molbev/msn172

Takagi Y, Kuramoto T, Voigt B et al (2009) An informative set of SSLP markers and genomic profiles in the rat MHC, the RT1 complex. Immunogenetics 61:189-197. https://doi.org/10.1007/ s00251-008-0352-9

Wang K, Li M, Hakonarson H (2010) ANNOVAR: Functional annotation of genetic variants from high-throughput sequencing data. Nucleic Acids Res 38:e164. https://doi.org/10.1093/nar/gkq603

Wang L, Mu Y, Xu L et al (2019) Genomic analysis reveals specific patterns of homozygosity and heterozygosity in inbred pigs. Animals 9:314. https://doi.org/10.3390/ani9060314

Waterhouse AM, Procter JB, Martin DMA et al (2009) Jalview Version 2-A multiple sequence alignment editor and analysis workbench. Bioinformatics 25:1189-1191. https://doi.org/10.1093/bioinforma tics/btp033

Waterston RH, Lindblad-Toh K, Birney E et al (2002) Initial sequencing and comparative analysis of the mouse genome. Nature 420:520-562. https://doi.org/10.1038/nature01262

Wickham H (2016) ggplot2: elegant graphics for data analysis. Springer-Verlag, New York

Yalcin B, Adams DJ, Flint J, Keane TM (2012) Next-generation sequencing of experimental mouse strains. Mamm Genome 23:490-498. https://doi.org/10.1007/s00335-012-9402-6

Yoshihara M, Saito D, Sato T et al (2016a) Design and application of a target capture sequencing of exons and conserved noncoding sequences for the rat. BMC Genom 17:593. https://doi. org/10.1186/s12864-016-2975-9

Yoshihara M, Saito D, Sato T et al (2016b) Application of a target capture sequencing of exons and conserved non-coding sequences to 20 inbred rat strains. BMC Genom 17:155-157. https://doi. org/10.1186/s12864-016-2975-9

Zerbino DR, Achuthan P, Akanni W et al (2018) Ensembl 2018. Nucleic Acids Res 46:D754-D761. https://doi.org/10.1093/nar/ gkx1098

Zhou Y, Zhou B, Pache L et al (2019) Metascape provides a biologistoriented resource for the analysis of systems-level datasets. Nat Commun 10:1523. https://doi.org/10.1038/s41467-019-09234-6

Publisher's Note Springer Nature remains neutral with regard to jurisdictional claims in published maps and institutional affiliations. 